\title{
Marcadores de aterosclerosis temprana y síndrome metabólico en niños
}

\author{
Salesa Barja ${ }^{1}$, Mónica Acevedo ${ }^{2}$, Pilar Arnaiz ${ }^{1}$, \\ Ximena Berríos ${ }^{3}$, Claudia Bambs ${ }^{3}$, Beatriz G uzmán $3 a$, \\ Jacqueline Carvajal2a, Berta Cassis ${ }^{3 a}$, Carlos Navarrete ${ }^{4 b}$. \\ Early markers for atherosclerosis \\ and metabolic syndrome in children
}

\begin{abstract}
Background: The high prevalence of obesity in children favors the appearance of metabolic syndrome (MS), increasing their cardiovascular risk. Aim: To evaluate components of MS in children and to correlate them with surrogate markers of atherosclerosis and subclinical inflammation. Material and methods: We studied 209 children aged $11.5 \pm 2$ years (50\% girls, 30\% prepuberal). Fifty percent had normal weight, 18\% were overweight, $29 \%$ were obese and 3\% were undernourished. A fasting blood sample was obtained to measure lipid levels, glucose, insulin, adiponectin and ultrasensitive C-reactive protein (usCRP). Subclinical atherosclerosis was evaluated using flow mediated dilatation of brachial artery (FMD) and carotid intima-media thicknes (IMT). For diagnosis of MS we adapted Cook's criteria. Results: Five percent of all children and $18 \%$ of those with overweight had MS. Children with more components had significantly higher fasting insulin and Homeostasis Model Assessment (HOMA) values. Clustering of MS components was also associated to higher values of usCRP and non significantly to lower adiponectin levels. We did not find differences in FMD. In obese children there was a tendency towards a higher IMT with clustering of MS components, although not significant. Conclusions: Children with overweight presented a higher risk of a clustering of MS components, which was also associated with insulin resistance and increase in ultrasensitive C reactive protein (Rev Méd Chile 2009; 137: 522-30).
\end{abstract}

(Key words: Atherosclerosis; Child; Metabolic syndrome)

Recibido el 16 de octubre, 2008. Aceptado el 19 de enero, 2009.

Trabajo financiado por el Centro de Investigaciones Médicas y por el Departamento de Pediatría (Concursos de Investigación 2006), de la Pontificia Universidad Católica de Chile.

${ }^{1}$ Departamentos de Pediatría, ${ }^{2}$ Enfermedades Cardiovasculares, y de ${ }^{3}$ Salud Pública. Facultad de Medicina. Pontificia Universidad Católica de Chile. Santiago, Chile. ${ }^{4}$ Programa de Magister en Estadísticas. Pontificia Universidad Católica de Chile.

aEnfermera Universitaria

${ }^{\mathrm{b}}$ Estadístico

Correspondencia a: Dra. Salesa Barja. Departamento de Pediatría. Pontificia Universidad Católica de Chile. Lira 85, 50 Piso, Santiago, Chile. Teléfono: 56 (2) 3543887, Fax: 56 (2) 6384307. E mail: sbarja@puc.cl 
E $34 \%$ de los escolares chilenos de primer año básico presenta exceso de peso ${ }^{1}$, prevalencia que aumenta en los niños mayores ${ }^{2}$, consecuencia de cambios en los hábitos de vida propios de nuestra sociedad en transición nutricional. En los adultos, a la obesidad y sedentarismo se suman otros factores de riesgo cardiovascular (FRCV), como la hipertensión arterial (HTA), diabetes mellitus tipo 2 y dislipidemias, lo cual explica que $28 \%$ de las muertes sean de causa cardiovascular ${ }^{3}$. En 1988 Reaven $^{4}$ describió el "Síndrome X" como la asociación de estas enfermedades con mayor RCV, postulando a la resistencia insulínica (RI) como mecanismo fisiopatológico común. Posteriormente ello ha derivado en una herramienta clínica para identificar pacientes con mayor riesgo. El año 2000 el Panel de Expertos del Programa Nacional de Educación en Colesterol, ATP III, adoptó el nombre de "Síndrome Metabólico", incorporando la obesidad abdominal como factor relevante ${ }^{5}$. Su prevalencia en adultos norteamericanos es de $38 \%{ }^{6}$ y en Chile se estima en alrededor de $22,6 \%{ }^{7}$.

En niños también han aumentado la presentación y agregación de FRCV asociados a obesidad ${ }^{8}$, se han adaptado los criterios de SM para adultos-11 y el año 2007 se publicó un consenso para la edad pediátrica ${ }^{12}$. En Estados Unidos de Norteamérica su prevalencia en adolescentes es de $4,2 \%$ y $9,2 \%{ }^{9-10}$ y en obesos de $38 \%$ y $50 \%{ }^{11}$. En Chile, se ha descrito en niños obesos, asociado a $\mathrm{R}^{13,14}$. La obesidad y los FRCV tienden a mantenerse en el tiempo, aumentando la morbimortalidad cardiovascular del adulto ${ }^{15}$, cuyo principal mecanismo fisiopatológico, la aterosclerosis (ATE), comienza en la niñez ${ }^{16}$.

En los últimos años han surgido técnicas ultrasonográficas no invasivas que detectan daño arterial precoz también en niños ${ }^{17-19}$ y se han estudiado marcadores plasmáticos de inflamación subclínica, asociados a ATE prematura y a mayor morbimortalidad cardiovascular ${ }^{20,21}$. Recientemente hemos comunicado que en niños la proteína c-reactiva ultra sensible (PCRus) se correlaciona al grado de obesidad 22 , sin demostrar daño funcional o estructural de la pared arterial ${ }^{23}$. Sin embargo, existe controversia en su relación con SM, tanto por desacuerdos en los criterios diagnósticos, como en los puntos de corte 24,25 .
El objetivo de la presente investigación fue estudiar la agregación de los componentes del SM en niños chilenos y establecer si de acuerdo a ello presentan evidencia de ATE precoz o de inflamación subclínica.

\section{PACIENTES Y MÉTOdo}

Se estudiaron 209 escolares de estrato socioeconómico medio y medio bajo, pertenecientes al área urbana de Santiago, 112 eran hijos o nietos de adultos participantes en un estudio epidemiológico previo, procedentes de una muestra de familias nucleares de distintas comunas de la ciudad de Santiago, elegidas por muestreo aleatorio, trietápico, estratificado por edad y situación socioeconómica, realizado entre octubre 2005 y diciembre de $2006^{22}$. Los restantes 97 fueron reclutados voluntariamente mediante cartas de invitación a los colegios de los primeros, ambos grupos fueron comparables en características socioeconómicas. Los criterios de inclusión fueron edad entre 6 y 15 años, ausencia de antecedente de: trastornos del metabolismo glucídico, dislipidemias, hipertensión, enfermedades metabólicas, infecciones o terapia farmacológica recientes y de enfermedad cardiovascular familiar. Se solicitó ayuno de $12 \mathrm{~h}$, el adulto responsable firmó un consentimiento informado, aprobado por el Comité de Ética de la Universidad.

Se midió peso y talla mediante balanza de palanca y estadiómetro SECA ${ }^{\circledR}$, con el niño descalzo, en ropa interior. Se calculó el índice de masa corporal $\left[\mathrm{IMC}=(\right.$ peso en $\left.\mathrm{kg}) /(\text { talla en } \mathrm{m})^{2}\right]$, expresado en percentiles (pc) y como puntaje z [ $\mathrm{z}=$ (valor medido - mediana) / 1DE]. Se definió obesidad como IMC $\geq$ pc95, sobrepeso: pc85-94, eutrofia: pc10-84 y bajo peso <pc10, referencia CDC-NCHS, 200026. Se midió el perímetro de cintura (PC) con cinta métrica inextensible, sobre el borde laterosuperior de la cresta ilíaca derecha, al final de una espiración, promediando dos mediciones. Se expresó referido al pc50 [PCp50 = (PC real/ideal) x 100] de un patrón internacional $^{27}$. Por último, se midieron pliegues cutáneos bicipital, tricipital (PTC), subescapular (PSE) y suprailíaco con un cáliper Lange ${ }^{\circledR}$, se usó la razón de PSE/PTC para estimar grasa troncal y se calculó el porcentaje de masa grasa mediante fórmulas de

ARtíCULO DE 
Slaughter ${ }^{28}$. La maduración sexual se evaluó según estadios de Tanner. Se midió PA según norma internacional ${ }^{29}$, con un equipo Dynamap Pro 100, Criticon".

La PCRus fue medida con nefelómetro Dade Behring BN II, con límite de detección 0,1 mg/L. La glicemia por método de glucosa-oxidasa y colesterol total (CT), colesterol HDL (CHDL) y triglicéridos (TG) por métodos enzimáticos estándar (analizador Yací), se calculó colesterol LDL (CLDL) mediante fórmula de Friedewald. La insulina plasmática se midió por quimioluminiscencia directa (Equipo Advia Centauro de Siemens) y la adiponectina por radioinmunoanálisis: (Kit Linco Research, St. Charles, MO, USA).

La RI se calculó mediante el índice de HOMA, Homeostasis Model Assesment Index ${ }^{30}$.

Para definir Síndrome Metabólico se adaptó la recomendación de $\mathrm{S} \mathrm{Cook}^{9}$, basada en los criterios para adultos del ATP III (Tabla 1), que considera la presencia de al menos tres componentes de los siguientes: Obesidad abdominal, PC $>\mathrm{pc} 90^{27}, \mathrm{CHDL} \leq 40 \mathrm{mg} / \mathrm{dl}, \mathrm{TG}>110 \mathrm{mg} / \mathrm{dl}, \mathrm{pc} 25$ y 75 de la población general ${ }^{31}$, glicemia $>100 \mathrm{mg} /$ $\mathrm{dl}^{31}$ y PAS o PAD $\geq$ Pc90, Task Force $2004^{29}$.

Estudios ultrasonográficos de aterosclerosis prematura: DMF se midió según recomendaciones internacionales 33 , con un equipo Hewlett Packard Sonos 5500, el niño en supino y el manguito en antebrazo no dominante, se escaneó la arteria braquial sobre el pliegue antecubital, midiéndose diámetro de íntima a íntima, con cáliper electrónico (software M'Ath $^{\circledR}$ Std). Se realizó la medición basal y después de la fase hiperémica reactiva, calculándose el porcentaje de dilatación. El grosor de la íntima-media carotidea (IMT) se midió según el consenso de Mannheim 2004 34 ; Con el mismo equipo se evalúa la pared posterior de cada carótida común, $1 \mathrm{~cm}$ proximal a su bifurcación. Las mediciones fueron realizadas off-line, por dos cardiólogas y una enfermera entrenada, en forma ciega al resultado de los exámenes de sangre.

El tamaño muestral de 209 niños se calculó de acuerdo a las prevalencias de obesidad y SM descritas, adecuado para encontrar el número necesario de niños con agregación de componentes de SM. Para el análisis descriptivo, se usó promedio y desviación estándar, para variables categóricas se usó análisis de varianza (Anova) y test exacto de Fisher para proporciones. Se consideró significativa una $p<0,05$. Se aplicó un modelo de regresión logística acumulativa para la prevalencia de uno, dos o más componentes de $\mathrm{SM}$, bajo el supuesto de Odds proporcionales, verificado por razón de verosimilitud.

\section{RESULTADOS}

Se estudiaron 209 niños de $11,5 \pm 2,2$ años de edad, $50 \%$ de sexo femenino, $30 \%$ prepúberes. Según estado nutricional, 3\% presentaba bajo peso, $50 \%$ eutrofia, $18 \%$ sobrepeso y $29 \%$ obesidad, no hubo diferencia entre estos grupos según edad, sexo o desarrollo puberal.

Tabla 1. C riterios diagnósticos de síndrome metabólico

\begin{tabular}{|lll|}
\hline Criterios & Adultos* & Niños** \\
\hline Perímetro de cintura & $>102$ cm (hombres) & $\geq$ percentil 90 \\
& $>88$ (mujeres) & $\geq$ percentil 90 \\
Hipertrigliceridemia $(\mathrm{mg} / \mathrm{dl})$ & $\geq 150$ & $\geq 110$ \\
Colesterol HDL bajo $(\mathrm{mg} / \mathrm{dl})$ & $\leq 40$ (hombres) & $\leq 40$ \\
& $\leq 50$ (mujeres) & $\leq 40$ \\
Presión arterial elevada & $\geq 130 / 85$ & $\geq$ percentil 90 \\
Glicemia elevada (mg/dl) & $\geq 110$ & $\geq 100$ \\
\hline
\end{tabular}

* NCEP-ATP III: National Cholesterol Education Program- Adult Treatment Panel III.

**Adaptado de S Cook ${ }^{9}$, ver referencias para cada criterio en el texto, sección Método. 
Considerando los componentes del SM, se encontró obesidad abdominal en 37,3\%, hipertrigliceridemia en $18,7 \%$, CHDL bajo en $12,4 \%$ y PA elevada en $8,6 \%$ de los niños, ninguno presentó glicemia elevada. Las frecuencias fueron mayores en los obesos $(95,2 \%, 37 \%, 21 \%$ y 9,7\%, respectivamente, Fisher: $\mathrm{p}<0,0001)$. No se encontró diferencia de acuerdo a sexo (femenino: $43 \%, 21 \%$, $14 \%$ y $7 \%$, masculino $32 \%, 16 \%, 10,5 \%$ y $10 \%)$ ni estado puberal (prepúberes: $28 \%, 16 \%, 8 \%$ y $8 \%$ vs púberes $41 \%, 20 \%, 14 \%$ y $9 \%), p>0,05$.

De acuerdo a la agregación de componentes del SM, 95 niños tenían cero, 79 tenían uno, 24 dos y 11 niños tenían tres, de modo que $5,2 \%$ ( $n=11 / 209$ ) cumplió con criterios de $\mathrm{SM}$. En el grupo de niños con sobrepeso, 18\% cumplía con dicha condición. Como se observa en la Figura 1, la agregación de componentes de SM se asoció al exceso ponderal ( $p$ $<0,0001$, modelo log-lineal con errores Poisson). En los niños sin componentes, ninguno era obeso, en aquellos con un componente
$44,3 \%$ era obeso, con 2 componentes $67 \%$ era obeso y todos los niños con SM eran obesos.

La Tabla 2 muestra las características generales según la agregación de componentes de SM: En el grupo total, no se encontró diferencia significativa en edad, sexo, ni estado puberal, aunque en los niños con SM hubo tendencia a menor proporción

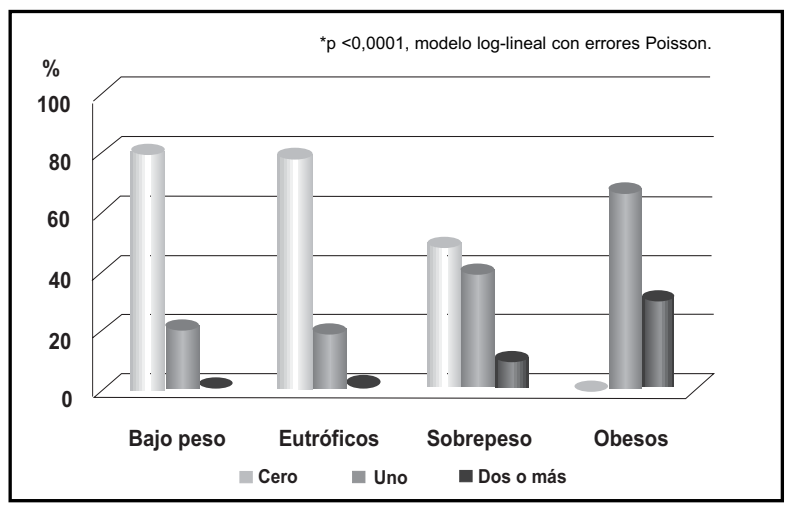

Figura 1. Agregación de componentes del Síndrome Metabólico de acuerdo al estado nutricional.

Tabla 2. Características clínicas de 209 niños, de acuerdo a agregación de componentes del síndrome metabólico (media \pm desviación estándar)

\begin{tabular}{|c|c|c|c|c|c|}
\hline \multirow[t]{2}{*}{ Medición } & \multicolumn{5}{|c|}{ Agregación de componentes del SM* } \\
\hline & Cero $(n=95)$ & Uno(n=79) & $\operatorname{Dos}(n=24)$ & Tres $(n=11)$ & p (Anova) \\
\hline Sexo femenino (\%) & 46,3 & 49,4 & 58,3 & 63,6 & ns \\
\hline Edad (años) & $11,42 \pm 2$ & $11,5 \pm 2$ & $11,9 \pm 2$ & $12,4 \pm 2,6$ & ns \\
\hline Prepúberes (\%) & 34,7 & 29,1 & 25,0 & 9,1 & ns \\
\hline Peso (Kg) & $40,5 \pm 12$ & $52,8 \pm 15$ & $58,8 \pm 16$ & $76,7 \pm 24$ & $<0,001$ \\
\hline Talla (cm) & $145,6 \pm 13$ & $148,8 \pm 14$ & $151,4 \pm 13$ & $159,7 \pm 14$ & $<0,01$ \\
\hline IMC $\left(\mathrm{kg} / \mathrm{m}^{2}\right)$ & $18,7 \pm 2,7$ & $23,7 \pm 4,4$ & $25,5 \pm 4,4$ & $29,4 \pm 4,5$ & $<0,001$ \\
\hline Score z IMC & $0,25 \pm 0,8$ & $1,3 \pm 1$ & $1,6 \pm 0,9$ & $2,1 \pm 0,3$ & $<0,001$ \\
\hline P. cintura $(\mathrm{cm})$ & $68,7 \pm 10$ & $83,2 \pm 12$ & $87 \pm 12$ & $101,5 \pm 15$ & $<0,001$ \\
\hline P. cintura $(\%)$ & $104 \pm 11$ & $126 \pm 19$ & $131 \pm 17$ & $149 \pm 16$ & $<0,001$ \\
\hline Masa grasa (\%) & $125 \pm 43$ & $205 \pm 94$ & $216,5 \pm 72$ & $259,3 \pm 92$ & $<0,001$ \\
\hline PSE/PTC (\%) & $81 \pm 26$ & $115 \pm 35$ & $129 \pm 33$ & $149 \pm 75$ & $<0,001$ \\
\hline PAS (mm Hg) & $101,8 \pm 7,6$ & $106,2 \pm 9,3$ & $103,7 \pm 12$ & $117 \pm 15$ & $<0,001$ \\
\hline PAD (mm Hg) & $55,3 \pm 5$ & $56 \pm 5,5$ & $56,02 \pm 5,3$ & $60,2 \pm 5,5$ & 0,038 \\
\hline
\end{tabular}

*Criterios diagnósticos para síndrome metabólico adaptados de $\mathrm{S} \mathrm{Cook}^{9}$.

IMC= Índice de masa corporal. Score z IMC= [(IMC real-IMC ideal $) / 1 \mathrm{DE})]$

$\mathrm{P}$ cintura $(\%)=\%$ de la Media para edad y sexo (27)

Masa grasa (\%) = Cálculo por fórmulas de Slaugther (28), expresada como \% de la Media.

PSE / PTC (\%) = Pliegue subescapular/Pliegue tricipital 
Tabla 3. Valores medios ( $\pm D E$ ) de lípidos plasmáticos, glicemia, insulinemia, índice de resistencia insulínica, PCRus, D M F e IMT en 209 niños, según el número de factores del síndrome metabólico

\begin{tabular}{|c|c|c|c|c|c|}
\hline \multirow{2}{*}{ Medición } & \multicolumn{5}{|c|}{ Agregación de componentes del SM* } \\
\hline & Cero $(n=95)$ & Uno $(n=79)$ & D os $(n=24)$ & Tres $(n=11)$ & $\begin{array}{r}p \\
\text { (Anova) }\end{array}$ \\
\hline Colesterol total $(\mathrm{mg} / \mathrm{dl})$ & $146,2 \pm 20$ & $149,8 \pm 28$ & $165,9 \pm 28$ & $150,5 \pm 28$ & $<0,01$ \\
\hline Colesterol HDL (mg/dl) & $57,3 \pm 10,3$ & $50,9 \pm 10,7$ & $46,6 \pm 11,4$ & $40,5 \pm 10,7$ & $<0,001$ \\
\hline Colesterol LDL (mg/dl) & $78 \pm 19$ & $85,7 \pm 23$ & $93,9 \pm 25.5$ & $85,7 \pm 22$ & $<0,01$ \\
\hline Triglicéridos (mg/dl) & $55,4 \pm 19$ & $66,9 \pm 30$ & $127 \pm 46$ & $122,6 \pm 19,6$ & $<0,001$ \\
\hline Glicemia (mg/dl) & $83,9 \pm 6$ & $84,9 \pm 4.6$ & $84,2 \pm 5$ & $83,4 \pm 5$ & ns \\
\hline Insulinemia (mU/ml) & $6,4 \pm 3,2$ & $10,5 \pm 7$ & $14,9 \pm 7,5$ & $18,1 \pm 10,5$ & $<0,001$ \\
\hline HOMA & $1,3 \pm 0,7$ & $2,2 \pm 1,5$ & $3,1 \pm 1,7$ & $3,7 \pm 2,2$ & $<0,001$ \\
\hline Adiponectina (mg/ml) & $17,5 \pm 5,9$ & $17,1 \pm 5,6$ & $15,3 \pm 4,1$ & $12,8 \pm 4,5$ & ns \\
\hline PCRus (mg/L) & $0,69 \pm 0,9$ & $1,75 \pm 3$ & $1,75 \pm 2$ & $1,82 \pm 2$ & $<0,01$ \\
\hline DMF (\%) & $9,3 \pm 3,8$ & $9,3 \pm 4,7$ & $9,3 \pm 4,3$ & $9,5 \pm 4$ & ns \\
\hline IMT media (mm) & $0,490 \pm 0,03$ & $0,486 \pm 0,03$ & $0,500 \pm 0,04$ & $0,486 \pm 0,02$ & ns \\
\hline
\end{tabular}

*Criterios diagnósticos para síndrome metabólico adaptados de S Cook ${ }^{9}$.

HOMA: Índice de resistencia insulínica (Homeostasis Model Assesment)

DMF: Dilatación de la arteria Braquial, mediada por flujo.

IMT: Grosor carotídeo miointimal

de prepúberes (Fisher: $\mathrm{p}>0,05)$. Al aumentar la agregación, se observó mayor peso, z IMC, z T/E, porcentaje de grasa total y grasa troncal (Anova, $p$ $<0,001)$. De acuerdo a los criterios diagnósticos de $\mathrm{SM}$, al agregar componentes se encontró mayor PC, PAS, PAD, TG y menor CHDL (Tablas 2 y 3).

En cuanto a los estudios de laboratorio (Tabla $3)$, con la agregación aumentaban insulinemia y HOMA (Anova, $p<0,001)$, CT y CLDL $(p<0,01)$ y aunque la adiponectina plasmática presentaba tendencia inversa, la diferencia no fue significativa. No se encontró diferencia en glicemia. Con respecto a los marcadores de ATE precoz, la PCRus aumentaba con la agregación $(p<0,01)$ y no se encontró diferencia para DMF o IMT ( $p>0,05)$.

Mediante correlación simple para marcadores de ATE, se encontró en el grupo total significación para CT y CLDL con PCRus. Mediante regresión logística acumulativa, los factores que predijeron la agregación de componentes del SM fueron $\mathrm{z}$ IMC (OR: 2,8, IC: 1,7-4,5) y HOMA (OR: 1,6, IC: $1,2-2,1)$ en forma conjunta, bajo el supuesto de odds proporcionales.

Con respecto a la agregación de componentes de SM, los niños con sobrepeso u obesidad ( $\mathrm{n}$ $=104$ ) presentaban similares tendencias descritas antes para el grupo total, tanto en sus características clínicas como exámenes de laboratorio. En los marcadores de ATE precoz, se observó una tendencia no significativa (Anova $\mathrm{p}=0,07$ ) de mayor IMT en aquellos con dos o más componentes de SM: $0,500 \pm 0,04$ vs $0,485 \pm 0,02$ y $0,481 \pm 0,02$ $\mathrm{mm}$, en aquellos con cero y un componente respectivamente (Figura 2). Se encontró asociación de IMT con triglicéridos ( $R=0,21$ ), Insulinemia $(\mathrm{R}=0,26)$ y HOMA $(\mathrm{R}=0,26)$, pero la asociación no se mantuvo al ajustar por otras covariables. Para el mismo grupo de niños, hubo correlación simple de PCRus con: ZIMC $(\mathrm{R}=0,48)$, $\% \mathrm{MG}(\mathrm{R}=0,48)$, grasa troncal $(\mathrm{R}=0,31)$ y $\mathrm{P}$ cintura $(\mathrm{R}=0,49), \mathrm{p}<0,001$. Hubo asociaciones más débiles con CLDL ( $R=0,25)$, CHDL ( $R=0,14$ ) y CT $(\mathrm{R}=0,18), \mathrm{p}<0,001$, las cuales también desaparecen al ajustar por otros factores, en análisis de regresión múltiple.

\section{Discusión}

En este estudio se analiza la presencia de marcadores de ATE precoz en niños, de acuerdo a la agregación de componentes del síndrome meta- 


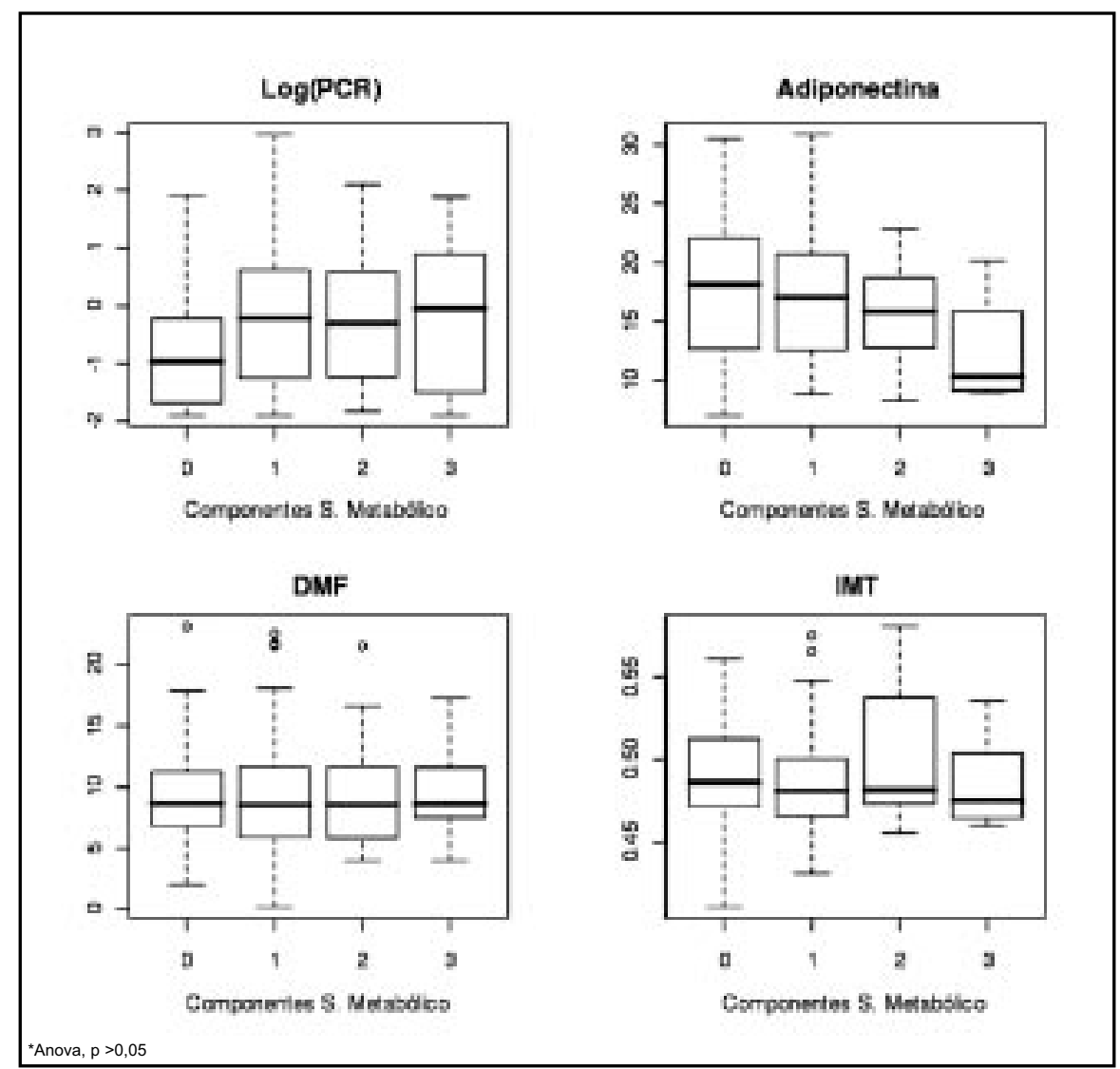

Figura 2. Marcadores de ATE precoz en 209 escolares, de acuerdo a agregación de componentes del síndrome metabólico.

bólico. Se demuestra que ella se asocia al grado de obesidad y de resistencia insulínica, al estado proinflamatorio y a una tendencia a menor adiponectina plasmática, factor protector cardiovascular. Los niños obesos con más de dos componentes de SM presentaban una tendencia a mayor grosor miointimal carotídeo, pero no encontramos disfunción endotelial. El trabajo de este grupo de investigación es pionero en nuestro país en la búsqueda de ATE precoz en niños.

La ocurrencia de 5,2\% SM encontrada es comparable a la prevalencia reportada por Cook, de 4,2\% en adolescentes de 12-19 años, en su mayoría pospúberes ${ }^{9}$. Nuestra muestra es de menor tamaño, edad y maduración puberal, pero tiene mayor representación de obesos que la población infantil chilena, $30 \%$ vs $19 \%^{2,1}$. En los niños obesos el 18\% cumple con criterios de SM, similar a $15,9 \%$ reportado en niños franceses e italianos ${ }^{35,36}$, pero menor a lo descrito en norteamericanos, de $39 \%$ y $29 \% 9,11$ y a $30 \%$ publicado en niños chilenos ${ }^{37}$. Ello se explica por factores como mayor edad, maduración o grado de obesidad en dichos estudios ${ }^{9,37}$, a la vez que posibles diferencias étnicas o raciales ${ }^{38}$. Por otra parte, si bien existe un consenso reciente para la definición de SM en niños y adolescentes ${ }^{12}$, hay disparidad en las prevalencias publicadas, por utilizarse diferentes criterios o puntos de corte $^{39}$. Hemos utilizado en nuestro estudio el criterio más aceptado y de sensibilidad adecuada para un estudio focalizado hacia la prevención cardiovascular $^{9}$.

La prevalencia de FRCV aislados en los obesos es comparable a otras ${ }^{8,35}$, aunque menor a la descrita en niños con mayor obesidad ${ }^{13,14}$. No

A RTíCULO DE 
encontramos casos con glicemia elevada, aun considerando el límite de $100 \mathrm{mg} / \mathrm{dl}$, propuesto recientemente ${ }^{32}$. Este marcador es poco sensible en niños, habiéndose planteado su reemplazo por el test de tolerancia oral a la glucosa, insulinemia 0 índices de $\mathrm{RI}^{11,39}$.

La obesidad abdominal fue el criterio más frecuente; el perímetro de cintura representa la grasa visceral, metabólicamente más activa, que a través de distintos mediadores favorecería la RI. Demostramos que ésta, en conjunto con la adiposidad, explican la agregación de componentes del $\mathrm{SM}^{13,38}$, en forma inversa, observamos que con la agregación disminuye la adiponectina, un marcador protector cardiovascular. Es notorio que también aumente el CLDL, un reconocido FRCV independiente ${ }^{8}$, correlacionado además con mayor grado de inflamación subclínica.

La elevación de PCRus se ha asociado a mayor morbimortalidad cardiovascular en adultos ${ }^{20} \mathrm{y}$ señala en niños un estado proinflamatorio que junto a factores pro-oxidativos pueden preceder al desarrollo de alteraciones de la pared endotelial $^{40,41}$. Hemos reportado antes en niños una asociación robusta entre grado de obesidad y PCRus $^{22}$, en el presente estudio ésta aumenta en niños que agregan componentes de SM, pero en forma dependiente de la adiposidad. Ford ${ }^{41}$ ha planteado que la obesidad abdominal es el factor principal que determina el estado proinflamatorio en adolescentes con SM, otros señalan que el IMC (grasa total) predice en forma significativa los cambios de PCRus ${ }^{42}$. En nuestro estudio, el perímetro de cintura es también un factor de peso en el mayor grado de inflamación asociada a la agregación de componentes de SM. Sin embargo, esto no ocurre en los obesos, a pesar que presentan mayor PC y zIMC; se podría plantear que a un mayor grado de inflamación basal, dependiente de la adiposidad, el sumar FRCV no aumenta este efecto, a diferencia de lo que ocurre en los eutróficos.

No se encontró evidencia de disfunción endotelial de acuerdo a agregación ni al grado de obesidad, pero se demostró una tendencia a mayor IMT en aquellos que teniendo exceso ponderal, agregan dos o más criterios de SM. En relación a estos marcadores, los estudios en niños han sido discordantes, pero existe mayor evidencia a favor de aumento de IMT asociada a SM, que de alteraciones funcionales. Según Reinher ${ }^{24}$, los criterios diagnósticos para SM se asocian en forma débil a IMT, el cual está más bien relacionado a intolerancia a la glucosa. Sin embargo, analiza niños con mayor obesidad y describe IMT de mayor magnitud y dispersión que los encontrados en nuestra muestra 0 en otros estudios ${ }^{18,19}$, lo cual podría deberse a diferencias raciales o metodológicas. Si bien se justifica realizar el test de tolerancia a la glucosa en niños con obesidad severa, no es aplicable como método de tamizaje para búsqueda de RCV. La insulinemia de ayunas pudiera serlo, ya que en nuestro estudio encontramos que en los obesos se asocia a IMT, hecho evidenciado también en niños obesos de Turquía $^{43}$ y en prepúberes italianos ${ }^{40}$, con mayor grosor miointimal y aumento de PCR, asociados a RI. Sin embargo, ambos estudios no analizan su relación a componentes de SM. Recientemente Aggoun ha planteado que DMF debiera ser considerado el marcador más precoz, asociada a HTA y no encuentra diferencias de IMT entre niños obesos y eutróficos ${ }^{25}$, pero tanto la baja magnitud de DMF como la diferencia según estado nutricional, no son comparables a otros ${ }^{18}$.

Frente a nuestros hallazgos y a la disparidad de la información disponible, podemos plantear que en niños con exceso de peso y estado proinflamatorio, es posible que se expresen en forma variable trastornos de la pared arterial, en la medida que se agreguen factores del SM. Es complejo aislar el efecto de la obesidad y probablemente en la población infantil existe una expresión gradual del proceso aterosclerótico en el tiempo, quizás con períodos críticos, modulada en forma paralela por factores genéticos, étnicos y ambientales.

El presente estudio colabora con el conocimiento de este proceso, aporta evidencia de que en los niños que agrupan componentes del SM existe un ambiente proinflamatorio y que si bien no presentan daño endotelial funcional, si además tienen exceso de peso, presentan tendencia al engrosamiento de la íntima arterial. Estudios de seguimiento, muestras de mayor tamaño y marcadores complementarios podrán ayudar a identificar cuáles niños iniciaron el proceso ATE, su posible reversibilidad y si el utilizar como herramienta clínica el SM puede ayudar a reconocer a aquellos con mayor riesgo cardiovascular futuro. 


\section{REFERENCIAS}

1. Junaeb.cl. Situación nutricional de los escolares de primero básico. 2007 (citado enero 2008). Disponible en: http://sistemas.junaeb.cl/estadosnutricionales 2007/index2.php

2. Muzzo S, Burrows R, Cordero J, Ramirez I. Trends in nutritional status and stature among school-age children in Chile. Nutrition 2004; 20: 867-72.

3. INE.cl. Instituto Nacional de Estadísticas de Chile (Citado 21 enero 2008). Disponible en: http://www.ine.cl/ canales/chile_estadistico/demografia_y_vitales/ estadisticas vitales/estadisticas vitales.phpwww.ine.cl

4. REAvEN GM. Banting lecture 1988. Role of insulin resistance in human disease. Diabetes 1988; 37: 1595-607.

5. Third Report of the National Cholesterol Education Program (NCEP) Expert Panel on Detection, Evaluation, and Treatment of High Blood Cholesterol in Adults (Adult Treatment Panel III) final report. Circulation 2002; 106: 3143-421.

6. Grundy SM, Brewer HB JR, CleEman JI, Smith SC JR, LENFANT C. Definition of Metabolic Syndrome: Report of the National Heart, Lung, and Blood Institute/ American Heart Association conference on scientific issues related to definition. Circulation 2004; 109: 433-8.

7. MaIz A. El síndrome metabólico y riesgo cardiovascular. Boletín de la Escuela de Medicina 2005; 30: 25-30. Disponible en http://escuela.med.puc.cl/ publ/boletin/20051/articulo4.pdf (Consultado el 2509-2008).

8. Freedman DS, Dietz WH, SRinivasan SR, Berenson GS. The relation of overweight to cardiovascular risk factors among children and adolescents: the Bogalusa Heart Study. Pediatrics 1999; 103: 1175-82.

9. Cook S, Weitzman M, Auinguer P, Nguyen M, Dietz WH. Prevalence of a metabolic syndrome phenotype in adolescents: findings from the third National Health and Nutrition Examination Survey, 19881994. Arch Pediatr Adolesc Med 2003; 157: 821-7.

10. De Ferranti SD, Gauvreau K, Ludwig DS, Neufeld EJ, Newburger JW, Rifai N. Prevalence of the Metabolic Syndrome in American Adolescents: Findings from the Third National Health and Nutrition Examination Survey. Circulation 2004; 110: 2494-7.

11. Weiss R, Dziura J, Burgert TS, Tamborlane WV, Taksali SE, YECKER CW, ET AL. Obesity and the metabolic syndrome in children and adolescents. N Engl J Med 2004; 350: 2362-74.

12. Zimmet P, Alberti G, Kaufman F, Tajima N, Silink M, Arslanian S, Wong G, Bennett P, Shaw J, Caprio S. On behalf of the International Diabetes Federation Task Force on Epidemiology and Prevention of Diabetes. The metabolic syndrome in children and adolescents. The Lancet 2007; 369: 2059-61.
13. Barja S, Arteaga A, Acosta AM, Hodgson Mi. Resistencia insulínica y otras expresiones del Síndrome Metabólico en niños obesos chilenos. Rev Méd Chile 2003; 131: 259-68.

14. Burrows R, Burgueño M, Leiva L, Ceballos X, Guimer I, Gattas V, et al. Perfil metabólico de riesgo cardiovascular en niños y adolescentes obesos con menor sensibilidad insulínica. Rev Méd Chile 2005; 133: 795-804.

15. FreEdman DS, Khan LK, DieTz WH, SRinivasan SR, BERENSON GS. Relationship of childhood obesity to coronary Heart disease risk factors in adulthood: the Bogalusa Heart Study. Pediatrics 2001; 108: 712-8.

16. Mathur Ks, Kumar V, Kashyap Sk. The Natural History of Coronary Atherosclerosis. Chest 1964; 46: 70-7.

17. Tounian $P$, Aggoun $Y$, Dubern B, VariLe V, GuY-Brand B, SIDI B, ET AL. Presence of increased stiffness of the common carotid artery and endothelial dysfunction in severely obese children: a prospective study. Lancet 2001; 358: 1400-4.

18. Woo KS, Chook P, Yu CW, Sung RY, Qiao M, Leung SS, ET AL. Overweight in children is associated with arterial endothelial dysfunction and intima-media thickening. Int J Obesity 2004; 28: 852-7.

19. Iannuzzi A, Licenziati MR, Acampora C, Salvatore V, AURIEMMA L, ROMANO ML, ET AL._Increased carotid intima-media thickness and stiffness in obese children. Diabetes Care 2004; 27: 2506-8.

20. Ridker PM, HenNekens Ch, Buring JE, Rifai N. Creactive protein and other markers of inflammation in the prediction of cardiovascular disease in women. N Engl J Med 2000; 342: 836-43.

21. Cook DG, Mendall MA, Whincup PH, Carey IM, BALAM L, MoRRIS JE, ET AL. C-reactive protein concentration in children: relationship to adiposity and other cardiovascular risk factors. Atherosclerosis 2000; 149: 139-50.

22. Acevedo M, Arnáiz P, Barja S, Bambs C, Berríos X, GuZMÁN B, ET AL. [Relationship of C-reactive protein to adiposity, cardiovascular risk factors and subclinical atherosclerosis in healthy children]. Rev Esp Cardiol 2007; 60: 1051-8.

23. Arnaíz P, Acevedo M, Barja S, Berríos X, C, Guzmán B, BAMBS C, ET AL. Arterioesclerosis subclínica, factores de riesgo cardiovascular clásicos y emergentes en niños obesos chilenos. Rev Chil Pediatr 2007; 78: 135-42.

24. Reinher T Wunsch R, De Sousa G, Toschre AM. Relationship between metabolic syndrome definitions for children and adolescents and intima-media thickness. Atherosclerosis 2007; 199: 193-200. Disponible en http://www.atherosclerosis-journal.com (Consultado el 6-01-2008)

25. Aggoun Y, Farpour-Lambert NJ, Marchand LM, Golay E, Maggio AB, BeghetTi M. Impaired endothelial and smooth muscle functions and arterial stiffness ap-

A RTÍCULO DE 
pear before puberty in obese children and are associated with elevated ambulatory blood pressure. European Heart Journal 2008; 29: 792-9. Disponible en http://eurheartj.oxfordjournals.org (Consultado el 21-5-2008).

26. BARLOW SE. Expert Committee. Expert committee recommendations regarding the prevention, assessment, and treatment of child and adolescent overweight and obesity: summary report. Pediatrics 2007; 120: S164-92.

27. Fernández J, Redden D, Pietrobeul A, Auison D. Waist circumference percentiles in nationally representative samples of african-american, european-american an mexican-american children and adolescents. J Pediatr 2004; 145: 439-44.

28. Siaughter MH, Lohman TG, Boileau RA, Horswill CA, StiLman RJ, VAn LoAn MD, ET AL. Skinfold equations for estimation of body fatness in children and youth. Hum Biol 1998; 60: 709-23.

29. National High Blood Pressure Education Program. Working Group on High Blood Pressure in Children and Adolescents. The Fourth report on the Diagnosis, Evaluation, and Treatment of High Blood Pressure in Children and Adolescents. Pediatrics 2004; 114: 555-76.

30. Matthews DR, Hosker JP, Rudenski AS, Naylor BA, TREACHER DF, TurNer RC. Homeostasis Model Assesment: insulin resistance and B-cell function from fasting plasma glucose and insulin concentrations in man. Diabetologia 1985; 28: 412-9.

31. NCEP. National Cholesterol Education Program Report of the Expert Panel on Blood Cholesterol Levels in Children and Adolescents, 1991. Bethesda, Md: National Heart, Lung, and Blood Institute Information Center; 1991.

32. Gabir MM, Hanson RL, Dabeifa D, Imperatore G, Roumain J, BENNETT PH, ET AL. The 1997 American Diabetes Association and 1999 World Health Organization criteria for hyperglycemia in the diagnosis and prediction of diabetes. Diabetes Care 2000; 23: 1108-12.

33. Corretti MC, Anderson TJ, Benjamin Ej, Celermajer D, Charbonneau F, Creager MA, et al. Guidelines for the ultrasound assessment of endothelial-dependent flow-mediated vasodilation of the brachial artery. A report of the International Brachial Artery Reactivity Task Force. J Am Coll Cardiol 2002; 39: 257-65.
34. Touboul $\mathrm{P}$, Hennerici Mg, Adams $\mathrm{H}$, Amarenco $\mathrm{P}$ Desvarieux M, Ebrahim S, et al. Mannheim IntimaMedia Thickness Consensus. Cerebrovasc Dis 2004; 18: 346-9.

35. Druet C, Dabbast M, Baltakse V, Payen C, Jourett B, BAUD C, ET AL. Insulin resistance and the metabolic syndrome in obese French children. Clin Endocrinol 2006; 64: 672-8.

36. Calcaterra V, Kiersy C, Muratori T, Telu S, Caramagna C, SCAGLa F, ET AL. Prevalence of metabolic syndrome (MS) in children and adolescents with varying degrees of obesity. Clin Endocrinol (Oxf) 2008; 68: 868-72.

37. Burrows R, Leiva L, Weistaub G, Ceballos X, Gattas V, LERA L, ET AL. Síndrome Metabólico en niños y adolescentes: asociación con sensibilidad insulínica y con magnitud y distribución de la obesidad. Rev Méd Chile 2007; 135: 174-81.

38. Cruz ML, Weigensberg MJ, Huang TT, Ball G, Shaibi GQ, Goran MI. The metabolic syndrome in overweight Hispanic youth and the role of insulin sensitivity. J Clin Endocrinol Metab 2004; 89: 108-13.

39. Goodman E, Daniels SR, Morrison JA, Huang B, Dolan LM. Contrasting prevalence of and demographic disparities in the WHO and NCEP ATP III definitions of metabolic syndrome among adolescents. J Pediatr 2004; 145: 445-51.

40. Giannini C, De Giorgis T, Scarinci A, Ciampani M, MarcovecChio ML, ChiareLi F, ET al. Obese related effects of inflammatory markers and insulin resistance on increased carotid intimae media thickness in pre-pubertal children. Atherosclerosis 2008; 197: 448-56.

41. Ford ES, AJANI UA, MOKDAD AH. The metabolic syndrome and concentrations of C-reactive protein among U.S. youth. Diabetes Care 2005; 28: 878-81.

42. Cardoso-SaldaÑa G, Juárez-Rojas JG, Zamora-GonZÁleZ J, Raygoza-Pérez M, Martínez-Alvarado R, PosadasSÁNCHEZ R, ET AL. C-reactive protein levels and their relationship with metabolic syndrome and insulin resistance in Mexican adolescents. J Pediatr Endocrinol Metab 2007; 20: 797-805.

43. Atabek ME, Pirgon O, KIVRaK AS. Evidence for association between insulin resistance and premature carotid atherosclerosis in childhood obesity. Pediatr Res 2007; 61: 345-9. 